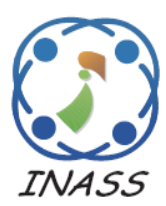

\title{
M2EW Algorithm for Increasing the Degree of Precision of Vertical Handover Network Selection
}

\author{
Zavli Juwantara $^{1} \quad$ Maman Abdurohman $^{1 *} \quad$ Sidik Prabowo $^{1}$ \\ ${ }^{1}$ School of Computing, Telkom University, Indonesia \\ * Corresponding author's Email: abdurohman@ telkomuniversity.ac.id
}

\begin{abstract}
This paper proposes the enhancement of a selected Fuzzy Multiple Attribute Decision Making algorithm, namely Modified Multiplicative Exponent Weighting (M2EW) algorithm. The main focus of improving the Multiplicative Exponent Weighting (MEW) algorithm is to enhance the reliability of alternative vector preference calculations for selecting network candidates in a vertical handover (VHO). However, MEW algorithm has resulted in relative accuracy from each network which has not, in many cases, matched the necessary conditions. The improvement is done by calculating the Euclidean distance between the nodes represented by the weight values of each selected network candidate parameter. There are various algorithms used to support the VHO mechanism, for example an algorithm that calculates the value of each of the network selection parameters such as RSS, bandwidth or network speed such as Simple Additive Weighting (SAW) and MEW algorithm. The result shows that the M2EW algorithm has increased the relative standard deviation value by $0.2 \%$ and has shown the same delay as the MEW algorithm.
\end{abstract}

Keywords: Vertical handover, Decision making algorithm, SAW, MEW, M2EW.

\section{Introduction}

Pervasive computing is a computational paradigm that has blended the existence of computers with the environment and hence it has become a natural part of the environment, such as wearable devices and monitoring systems [1]. The existence of pervasive computing for now cannot be separated from the existence of the Internet of Things (IoT). By utilizing IoT, it is possible for any existing object to sense and control other objects remotely within the entire existing Internet network, so as to integrate between the real world and the digital world [2].

As times progressed, the Next Generation Wireless System concept has emerged as a concept of the generation of wireless networks that already support Vertical Handover (VHO) mechanism, which enables users to maintain internet connections when network transitioning. The concept can be used as a solution of one of the IoT utilization problems in pervasive computing such as the amount of cost required when using the cellular network, and the minimum coverage area when using Wireless Local Area Network (WLAN). The necessary VHO mechanism for the device is therefore able to maintain its internet connection.

There are many VHO decision algorithms that have developed, for example SAW and MEW algorithms [3], the two of which are a combined algorithm hence uses various parameters to perform the VHO process.

From previous research using simulations of four different classifications, i.e. conversational, streaming, interactive and background, it has been found that SAW and MEW algorithms have good performances in those four classifications with the following values: $92.36 \%, 96.47 \%, 98.44 \%$ and $98,84 \%$ [3].

In this paper, we have proposed the new algorithm on pervasive computing in the form of a fall detector for the elderly, which is a wearable device that must be connected to the internet to send and process data obtained by the device and carry out improvements in the performance of the algorithm that has the best relative standard deviation value in order to obtain better reliability. The VHO decision algorithms to be implemented are the SAW, MEW algorithm; also, the best modified algorithm 
mentioned is included in the best VHO decision algorithm. The main focus in enhancing this selected algorithm is to enhanced the reliability of the alternative vector preference calculations for the selecting network candidates in a vertical handover mechanism based on increasing the degree of percision of vertical handover network selection.

\section{Related Works}

There are various existing vertical handover decision algorithms, and there are four main approaches used as vertical handover decision algorithms as follows: (1) RSS based, (2) bandwidth based, (3) cost-function based, and (4) combined algorithm based approaches [4]. (1) RSS based is an algorithm which use Received Signal Strength as the main parameter. So in this algorithm has the asvantage of the lack of occurence of failure on handover made by the handover that is not necessary so that the formation of damage to the connection but has a drawback regarding packet delay probability that is up to $1 \%$, there is effort to reduce by adjusting ASST[4], [5]. (2) Bandwidth based is an algorithm which used bandwidth as the main parameter on doing the handover mechanism. So, in this algorithm allows the "ping-pong" effect and also this algorithm has a high handover failure probability without considering the RSS. (3) Cost-function based is an algorithm which based on cost-fucntion for the network combination such as RSS, coverage area network, bandwidth allocation, cost, reability, and security. The advantage of algorithm is in the delay of the handover decision is reduced, so reducing the blocking handover and high troughput value. But it makes reducing the reability such as the difficulty of parameters measurement. (4) Combination algorithm based approach is an algorithm which used combined various parameters in taking a handover on the handover mechanism as well as cost-function based. By using algorithm, it can improve the performance by reducing unnecessary handover and "ping-pong" effect [4]

The VHD algorithm based on the combination algorithm is a high reliability algorithm because it has a training algorithm system like Multiple Attribute Decision Making algorithm. It is a combined algorithm that uses various parameters simultaneously such as artificial neural network and fuzzy logic [6].

Fuzzy logic is one of the concepts on soft computing commonly used in uncertainty conditions provided with selected information. It usually has a more prioritized level of truth than a binary classification that is generally "true" and "false" [7].
In a previous study [3], the simulation applied different classifications which are conversational, streaming, interactive and background, using three algorithms namely SAW, MEW, and Gray Relational Analysis (GRA) algorithms. The obtained results of SAW and MEW algorithms show the bestperforming algorithm of the classifications with the following values: $92.36 \%$ for the conversational class, $96.36 \%$ for the streaming class, $98.44 \%$ for the interactive class and $98.84 \%$ for the background class.

Another study [8] is about vertical handover decision process for mobile devices that also requires determination by taking into calculation delay, bandwidth, cost and jitters. In this study SAW and MEW methods have been selected to determine the use of device connectivity to Wi fi and WiMax. From the result of the research, MEW method has shown a better determination result of $35,75 \%$ than that of SAW method, which is $12,64 \%$.

This paper discusses improvement of MEW algorithm as the best algorithm of both algorithms implemented in the wearable device developed, i.e. the fall detector for the elderly.

\subsection{Vertical handover}

Vertical Handover (VHO) is a concept of wireless networking that allows users to maintain connections within a network when transitioning from one to another network [9]. The Next Generation Wireless System is an integration of wireless access technology that is heterogeneous. In the process, VHO has three main processes: (1) System discovery process: in this process the terminal on the device is equipped with several interfaces that must determine which network to use and what services are there on the network. (2) VHO decision process: in this process the device will determine which network to target. The decision is taken on the parameters including delay, power and user preferences. (3) VHO execution process: in this execution process, the connection status must be smoothly re-directed from the network used to the new network. [10].

\subsection{Simple additive weighting algorithm}

SAW algorithm is based on fuzzy issues. Fuzzy logic acts as inappropriate information on some user attributes and preferences. In fuzzy MADM there are two steps that convert fuzzy data to real numbers. The second step uses the classical MADM method in determining the ranking of the network candidates, one of which is SAW algorithm. 
In the SAW algorithm, the overall value of each network candidate will be determined based on the weight of each attribute. Each value of the network candidate $i$ is derived from adding the contribution of the normalization of each $r_{j}$ matrix multiplied by the weight of the weight interest assigned $w_{j}$ from the matrix $j$. It will then select the selected $A_{S A W}{ }^{*}$ network based on

$$
A_{S A W}^{*}=\arg \max _{1 \in M} \sum_{j=1}^{N} w_{j} r_{i j}
$$

where $N$ indicates parameters and $M$ indicates the network candidates targeted by the device [6].

\subsection{Multiplicative exponent weighting algorithm}

In MEW algorithm, VHO makings are showed in a matrix form which has $i$ variable expresses network candidate and $j$ variable expresses to the attributes. Thus, $S_{M E W}$ value is depended by the weight of the product of each attribute or matrix [3].

$$
S_{i}=\prod_{j=1}^{N} x_{i j}^{w j}
$$

where $x_{i j}$ is the marker of the variable $j$ expresses the candidate network $i$, and $w_{j}$ expresses the weight of attribute $\mathrm{j}$ and $\sum_{j=1}^{N} w_{j}=1 w_{j}$ is a positive value for the benefit matrix and a negative value for the cost matrix [3]. The benefit matrix is said to have the best value when it has a big value; on the contrary, the cost matrix is said to have the best value when it has a small value. Therefore, the $R_{M E W}$ ratio value between $i$ can be calculated as follows.

$$
R_{M E W}=\frac{\prod_{j=1}^{N} x_{i j}^{w_{j}}}{\prod_{j=1}^{N}\left(x_{i j}^{* *}\right)}
$$

The equation is $0 \leq \mathrm{Ri} \leq 1$, and therefore in the selection of networks the equation $A_{M E W^{*}}$ is used.

$$
A_{M E W}^{*}=\arg \max _{1 \in M} R_{M E W}
$$

The focus is the weight of the $w_{j}$ needed for MEW. The value of the weight depends on the needs of the QoS of the existing traffic class [3].

\subsection{Euclidean distance}

Any fluctuating or uncertain weight has value information that is referred to as the coordinate point $(x, y)$ in the Cartesian plane. Information changes of each parameter value is represented in two- dimensional coordinates $(x, y)$ such as $S 1=(x 1, y 1)$ and $S 2=(x 2, y 2)$.

Thus, the calculation of the euclidean distance values between the two points above has the coordinate variables $(x, y)$ that can be calculated by the equation:

$$
d=\sqrt{\sum_{i=1}^{n}\left(S_{1}-S_{2}\right)}
$$

where $S_{1}=$ value of $S_{1}, S_{2}=$ value of $S_{2}, d=$ value of the distance between $S_{1}$ and $S_{2}$ [11].

\subsection{System design}

The design of this system architecture encompasses the entire system parts such as the design of the mechanism of the vertical handover itself and the wearable devices used in the system.

As already described, in this paper, the prototype is a wearable device for fall detection for the elderly. Fig. 1 is a block diagram of an elderly fall detector architecture that will be used to perform vertical handover simulations by using developing algorithms.

Based on Fig. 1, the simulation process is performed using the elderly fall detection device. As already explained, the vertical handover process begins with the system discovery process to determine what networks are available and to find out what services are there on the network. Then it is followed by $\mathrm{VHO}$ decision process using the select SAW algorithm (first algorithm), MEW algorithm (second algorithm) and M2EW algorithm (third algorithm and the algorithm proposed in this

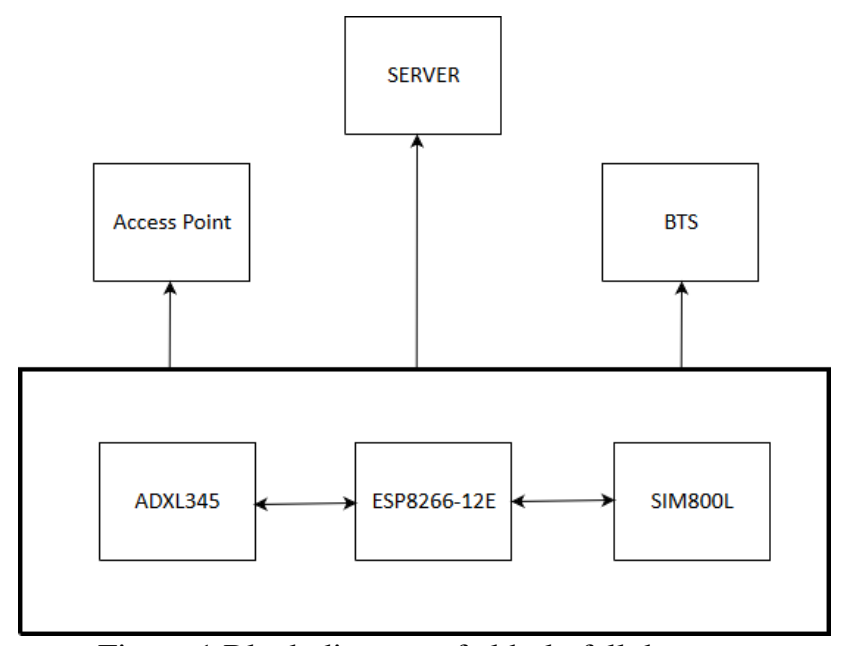

Figure.1 Block diagram of elderly fall detector 


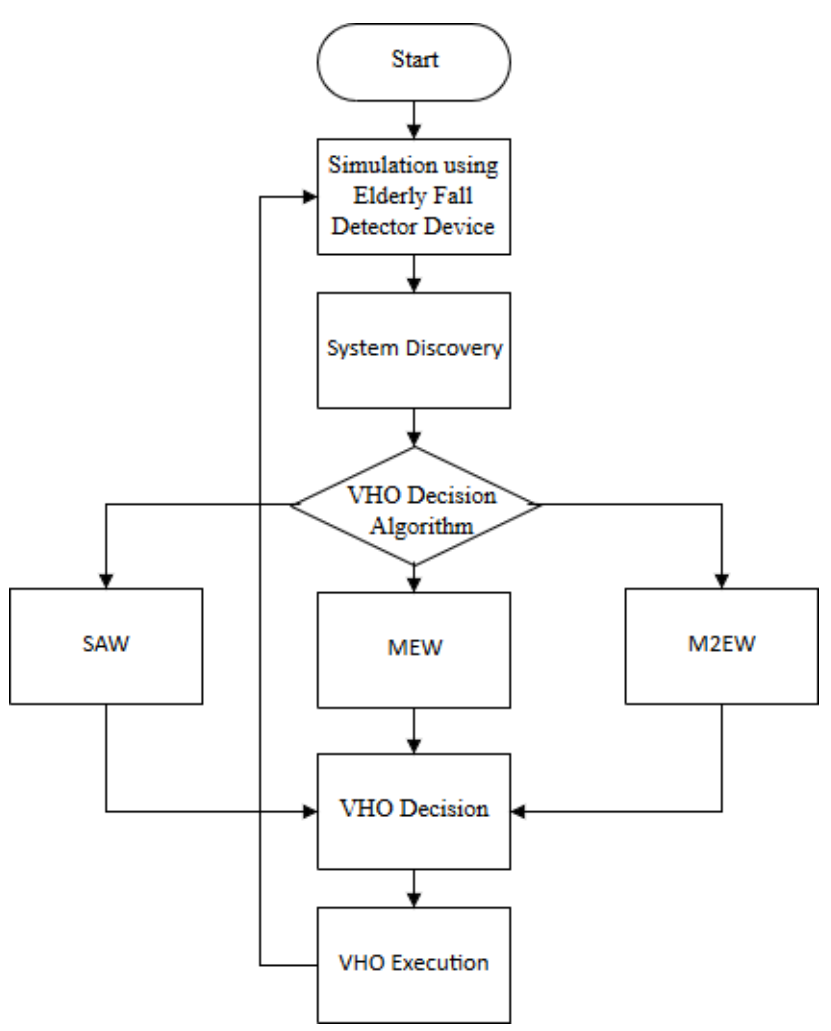

Figure.2 Flowchart of vertical handover process

research). After determining which network will be addressed, VHO execution process will be conducted to perform the mechanical vertical handover destination network as illustrated in Fig. 2.

\section{Modified-multiplicative exponent weighting algorithm}

\subsection{Euclidean weight value}

Calculating the Euclidean distance between nodes/networks by taking into account the vector values, bandwidth and network speed in this study is referred to as Euclidean weight value (EWV). EWV calculations are used to calculate alternate preference vector values to decide the ranking of each available network candidate in the area.

The value of each node/network vector is denoted as follows.

$$
S_{i}=\prod_{j=1}^{N} x_{i j}^{w j}
$$

Where, $S_{i}=$ Vector value of the network candidate $i$, $x_{i j}=$ Parameter value of attribute $j$ from network candidate $i, w_{j}=$ Weight value of attribute $j \sum_{j=1}^{N} w_{j}=1$.

After obtaining the information on the vector value of each candidate network, calculation will be made to assess the selected network by calculating the alternative preference vectors in the determination of the network to be used which is as follows.

$$
V_{i}=\frac{S_{i}}{\sqrt{\left(S_{i}-S_{j}\right)^{2}}}
$$

where $V_{i}=$ alternative vector calculation value, $S_{i}=$ vector value of the first network parameter, $S_{j}=$ vector value of the second network parameter.

\subsection{M2EW algorithm design}

The M2EW algorithm is a modified algorithm of MEW algorithm hence having different sections from the MEW algorithm, i.e. utilizing Euclidean distance calculations. The following is a network determination step when the vertical handover decision mechanism uses M2EW algorithm : (1) the M2EW algorithm begins with a weighted improvement on each benefit matrix and cost matrix by using the equation, $w_{i}=\frac{b_{i}}{\sum_{i=1}^{N} w_{i}}$, (2) the initial weight of the criterion $w$ is the weight value set as the weight percentage of each network parameter to be selected, which consists of bandwidth, RSS, network speed, (3) Total weight $\sum_{i=1}^{N} w_{i}=1$, (4) Vector $S_{i}$ is calculated by the criteria $j$ of each node/network alternative that is raised with the node/network (Eq. 6), (5) It is this alternative ranking that serves as the modifying phase of M2EW algorithm against MEW algorithm. In the MEW algorithm, alternative ranking of network candidates is performed by calculating vector $V_{i}$ where each vector $S_{i}$ is divided by total number of vector $S_{i}$, whereas in the M2EW, the calculation of vector $S i$ is divided by the Euclidean Weight Value of each vector $S$, which is an alternative preference of vector $S_{i}$.

Each node/network alternative has a value of $V_{i}$ which is used for reference rankings of available networks to be selected in a vertical handover decision mechanism. It can therefore be determined that the alternative node with the largest $V_{i}$ is the network to be selected to perform the vertical handover mechanism.

\section{Numerical Example}

This section will explain how the SAW, MEW and M2EW algorithms work numerically in outlining the vertical handover decision algorithm in the select network used in this study. As an example, there is a mobile terminal currently connected to WLAN and cellular networks that have to make decisions between both $\mathrm{A} 1$ and $\mathrm{A} 2$ network candidates, where A1 is WLAN and A2 is the cellular network. In 
selecting the decision for the vertical handover in this study, bandwidth, RSS and network speed are used and denoted by $\mathrm{X} 1, \mathrm{X} 2$ and $\mathrm{X} 3$. Then, the decision matrix used is as follows:

$$
\mathrm{D}=\begin{array}{|l|l|l|l|}
\hline & \mathrm{X} 1 & \mathrm{X} 2 & \mathrm{X} 3 \\
\hline \mathrm{A} 1 & 0.8 & 0.7 & 0.35 \\
\hline \mathrm{A} 2 & 0.7 & 0.6 & 0.8 \\
\hline
\end{array}
$$

The vertical handover criteria preference will be modelled as the weight assigned by the user. The $\mathrm{W}$ data are shown in Eq. (8).

$$
w_{d}=\left[\begin{array}{lll}
0.3 & 0.3 & 0.4
\end{array}\right]
$$

The vertical handover decision algorithm in this numerical example section will use the data above. The next section will discuss how the confusion of SAW, MEW and M2EW algorithms will be applied.

\subsection{Simple additive weighting algorithm}

The SAW algorithm requires a comparable scale for all elements that is used to normalize the values that serve as the factor that determines the benefit or cost criteria. The criteria for benefit is that the biggest value is the best alternative; on the contrary, for the cost criteria, the smallest is the best. In this scheme $x_{i j}$ is an alternative of score performance $A_{i}$ that concerns the criteria value of $x_{j}$.

$\mathrm{D}^{\prime}=\quad$\begin{tabular}{|c|c|c|c|}
\hline & $\mathrm{X} 1$ & $\mathrm{X} 2$ & $\mathrm{X} 3$ \\
\hline $\mathrm{R} 1$ & 1 & 0.875 & 1 \\
\hline $\mathrm{R} 2$ & 0.875 & 0.75 & 1.33 \\
\hline
\end{tabular}

Then, based on the weight value used in Eq. (8), the value of the velocity obtained based on the matrix $\mathrm{D}$ is

$$
R_{S A W}=\left[\begin{array}{ll}
0.9265 & 1.02083
\end{array}\right]
$$

After the value for matrix $\mathrm{R}$ is obtained, the ranking of the network candidate on the matrix $R$ is performed.

$$
A_{S A W}^{*}=[1.02083]
$$

Based on the ranking resulted from Eq. (9), the obtained biggest value can be seen in Eq. (10), which is the biggest value of matrix $\mathrm{R}$ and therefore in the vertical handover execution phase, the selected network is A2.

\subsection{Multiplicative exponent weighting algorithm}

MEW algorithm is deemed a dimensionless analysis because the mathematical structure of this algorithm omits any measurement unit. Transformation is not required when using multiplication between attribute values used. The weights will become the values of the exponent associated with each attribute value. Eq. (8) is the equation for weight to be used for $A_{M E W}$.

$$
\begin{gathered}
S_{i}=\left[\begin{array}{ll}
0 & 0.7
\end{array}\right] \\
R_{M E W}=\left[\begin{array}{ll}
0.44 & 0.56
\end{array}\right] \\
A_{M E W}^{*}=\left[\begin{array}{l}
0.56
\end{array}\right]
\end{gathered}
$$

Similar to that of SAW algorithm, the result of using MEW algorithm shows that the select network is $\mathrm{A} 2$.

\subsection{Modified-multiplicative weighting algorithm}

The following is calculation result using M2EW algorithm.

$$
\begin{gathered}
S_{i}=\left[\begin{array}{ll}
0 & 0.7
\end{array}\right] \\
R_{M 2 E W}=\left[\begin{array}{ll}
3.61 & 4.61
\end{array}\right] \\
A_{M 2 E W}^{*}=[4.61]
\end{gathered}
$$

The result of using M2EW algorithm shows that the select network is A2.

\section{Results and Discussion}

Our scenario is depicted on Fig. 3 that explains a cell coverage area comprises WLAN area and Cellular (GPRS) area. The device is intended to connect the suitable network. The test is aimed to find out the performance of the algorithm by performing calculations against the RSD of the work process on the algorithm. RSD is the standard dispersion of the probability distribution or the frequency distribution.

$$
R S D=\frac{S}{X} \times 100 \%
$$

In many cases the relative standard deviation is used that expressed in percentage [12]. Based on Table 1 and Fig. 4, it can be conclude that MEW algorithm has a higher deviation standard than SAW algorithm.

Based on the value of RSD obtained, i.e. $6.8 \%$ for SAW algorithm and $16.9706 \%$ for MEW algorithm, it can be seen that RSD of MEW algorithm is better than that of SAW algorithm. 


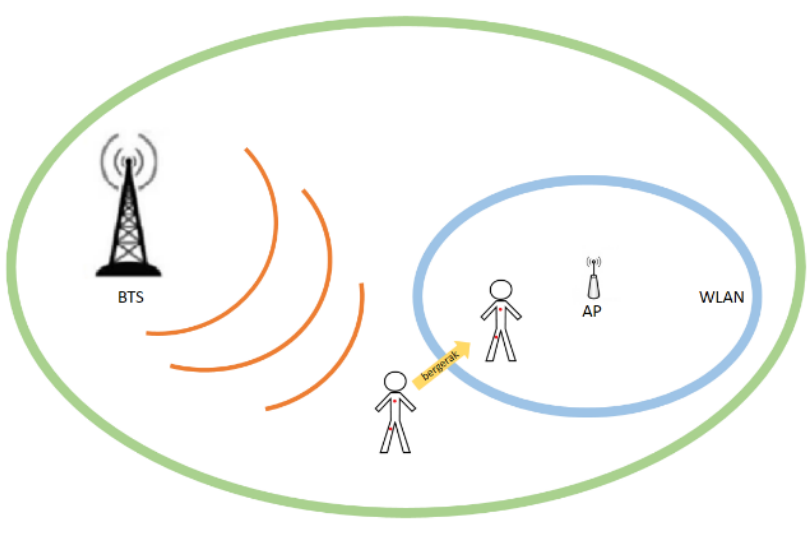

Figure.3 Scenario of the vertical handover

\subsection{Comparison of QoS delay parameters on SAW and MEW algorithms}

After testing the vertical handover decision algorithm using RSD, we conducted a performance test of vertical handover decision algorithm on the delay that has happened. Delay is the time it takes to transmit data from the source node to the destination node. Fig. 5 and Table 2 show the results of the tests of SAW algorithm and MEW algorithm on the wearable device for elderly fall detection in terms of delay.

Based on testing using the elderly fall detector device that implemented the vertical handover mechanism, the performance of MEW algorithm has smaller delay than SAW algorithm. This can be seen from the smaller average delay experienced by the MEW algorithm in sending data from the wearable device elderly fall detector, which is about 26 seconds; in comparison, the required time for using SAW algorithm is about 29 seconds.

Table 1. RSD value of SAW and MEW algorithms

\begin{tabular}{|c|c|c|}
\hline & SAW & MEW \\
\hline V1 & 0.9625 & 0.44 \\
\hline V2 & 1.02083 & 0.56 \\
\hline S & 0.066701 & 0.08 \\
\hline X & 0.973665 & 0.5 \\
\hline RSD & $6.850547 \%$ & $16.9706 \%$ \\
\hline
\end{tabular}

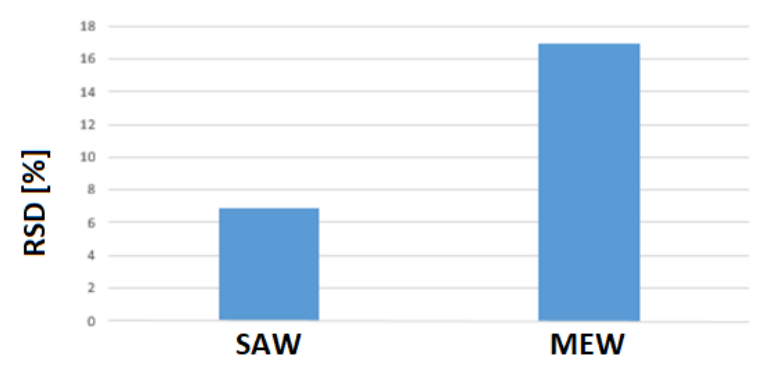

Figure.4 Comparison of RSD value between SAW and MEW algorithms

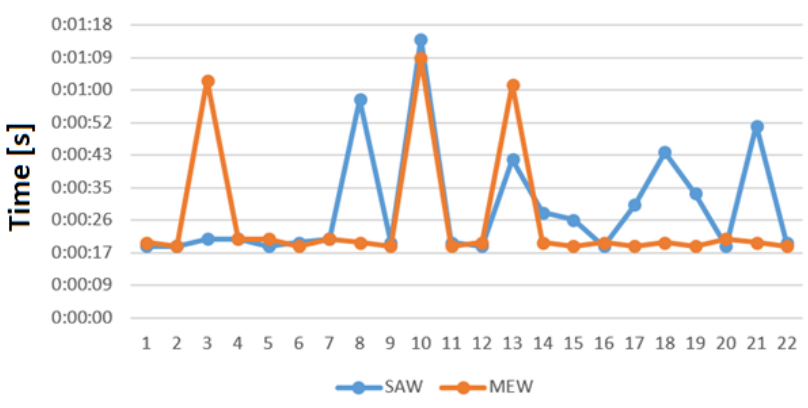

Figure.5 Comparison of delay between SAW and MEW algorithms

Table 2. Delay values of SAW and MEW algorithms

\begin{tabular}{|c|c|c|}
\hline $\begin{array}{c}\text { Transmission } \\
\text { No. }\end{array}$ & $\begin{array}{c}\text { SAW } \\
\text { (second) }\end{array}$ & $\begin{array}{c}\text { MEW } \\
\text { (second) }\end{array}$ \\
\hline 1 & $0: 00: 19$ & $0: 00: 20$ \\
\hline 2 & $0: 00: 19$ & $0: 00: 19$ \\
\hline 3 & $0: 00: 21$ & $0: 01: 03$ \\
\hline 4 & $0: 00: 21$ & $0: 00: 21$ \\
\hline 5 & $0: 00: 19$ & $0: 00: 21$ \\
\hline 6 & $0: 00: 19$ & $0: 00: 19$ \\
\hline 7 & $0: 00: 20$ & $0: 00: 21$ \\
\hline 8 & $0: 00: 21$ & $0: 00: 20$ \\
\hline 9 & $0: 00: 58$ & $0: 00: 19$ \\
\hline 10 & $0: 00: 20$ & $0: 01: 09$ \\
\hline 11 & $0: 01: 14$ & $0: 00: 19$ \\
\hline 12 & $0: 00: 19$ & $0: 00: 20$ \\
\hline 13 & $0: 00: 42$ & $0: 00: 19$ \\
\hline 14 & $0: 00: 28$ & $0: 00: 20$ \\
\hline 15 & $0: 00: 26$ & $0: 00: 19$ \\
\hline 16 & $0: 00: 19$ & $0: 00: 20$ \\
\hline 17 & $0: 00: 30$ & $0: 00: 19$ \\
\hline 18 & $0: 00: 44$ & $0: 00: 20$ \\
\hline 19 & $0: 00: 33$ & $0: 00: 19$ \\
\hline 20 & $0: 00: 19$ & $0: 00: 21$ \\
\hline 21 & $0: 00: 51$ & $0: 00: 20$ \\
\hline 22 & $0: 00: 20$ & $0: 00: 19$ \\
\hline Average & $0: 00: 29$ & $0: 00: 26$ \\
\hline & & \\
\hline
\end{tabular}

Based on the results, both in terms of RSD on the algorithm and delay that has occurred on the wearable devices, it can be concluded that MEW is suitable algorithm to use in the execution of vertical handover on wearable devices compare to SAW. In this paper, therefore, proposed method modified the MEW (M2EW) algorithm to improve the performance of the algorithm by calculating Euclidean distance during the calculation phase of alternative network preferences as described in the previous chapter.

\subsection{Test result of using MEW and M2EW algorithms}

Using the same sample data, the MEW and M2EW algorithms tests resulted showed in the Table 3 and Fig. 6. Based on the value of RSD obtained, 
i.e. $16.9706 \%$ for MEW algorithm and $17.20454 \%$ for M2EW algorithm.

The result shows that proposed method M2EW has better RSD value compare to MEW by approx. $0.2 \%$. Thus, it can be concluded that M2EW algorithm is a better recommendation for using in the wearable device elderly fall detectors.

\subsection{Comparison of QoS delay parameters of MEW and M2EW algorithms}

Like Fig. 5 and Table 2, Fig. 7 and Table 4 show the test results of the MEW and M2EW algorithms in term of the delay traffic using the elderly fall detector device. It shows that the performance of MEW and M2EW algorithms have, relatively, similar delay. It is evident from the average delay duration by MEW and M2EW algorithms in sending data from the wearable device for elderly fall detection, which is about 26 seconds.

Table 3. RSD value of MEW and M2EW algorithms

\begin{tabular}{|c|c|c|}
\hline & MEW & M2EW \\
\hline V1 & 0.44 & 3.61 \\
\hline V2 & 0.56 & 4.61 \\
\hline S & 0.08 & 0.707107 \\
\hline X & 0.5 & 4.11 \\
\hline RSD & $16.9706 \%$ & $17.20454 \%$ \\
\hline
\end{tabular}

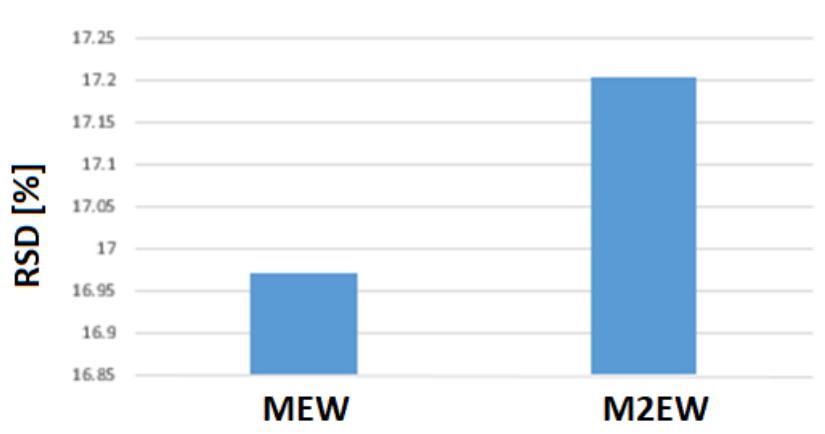

Figure.6 Comparison of RSD values using MEW and M2EW algorithms

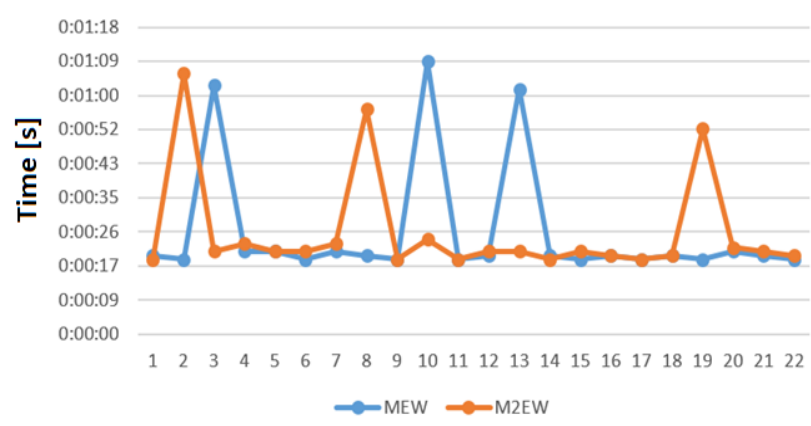

Figure.7 Comparison of delay traffic between MEW and MSEW algorithms
Table 4. Delay values of MEW and M2EW algorithms

\begin{tabular}{|c|c|c|}
\hline Transmission no. & $\begin{array}{c}\text { MEW } \\
\text { (second) }\end{array}$ & $\begin{array}{c}\text { M2EW } \\
\text { (second) }\end{array}$ \\
\hline 1 & $0: 00: 20$ & $0: 00: 19$ \\
\hline 2 & $0: 00: 19$ & $0: 01: 06$ \\
\hline 3 & $0: 01: 03$ & $0: 00: 21$ \\
\hline 4 & $0: 00: 21$ & $0: 00: 23$ \\
\hline 5 & $0: 00: 21$ & $0: 00: 21$ \\
\hline 6 & $0: 00: 19$ & $0: 00: 21$ \\
\hline 7 & $0: 00: 21$ & $0: 00: 23$ \\
\hline 8 & $0: 00: 20$ & $0: 00: 57$ \\
\hline 9 & $0: 00: 19$ & $0: 00: 19$ \\
\hline 10 & $0: 01: 09$ & $0: 00: 24$ \\
\hline 11 & $0: 00: 19$ & $0: 00: 19$ \\
\hline 12 & $0: 00: 20$ & $0: 00: 21$ \\
\hline 13 & $0: 00: 19$ & $0: 00: 21$ \\
\hline 14 & $0: 00: 20$ & $0: 00: 19$ \\
\hline 15 & $0: 00: 19$ & $0: 00: 21$ \\
\hline 16 & $0: 00: 20$ & $0: 00: 20$ \\
\hline 17 & $0: 00: 19$ & $0: 00: 19$ \\
\hline 18 & $0: 00: 20$ & $0: 00: 20$ \\
\hline 19 & $0: 00: 19$ & $0: 00: 52$ \\
\hline 20 & $0: 00: 21$ & $0: 00: 22$ \\
\hline 21 & $0: 00: 20$ & $0: 00: 21$ \\
\hline 22 & $0: 00: 19$ & $0: 00: 20$ \\
\hline Average & $0: 00: 26$ & $0: 00: 26$ \\
\hline & & \\
\hline & & \\
\hline & & 0
\end{tabular}

Based on the two parameters used in the test, it shows that M2EW algorithm performed is deemed successful. It has been proven by the increase in the value of the RSD of $0.2 \%$ and the resulted delay that is relatively similar compare to MEW algorithm.

\section{Conclusion}

Based on the tests conducted, the three vertical handover decision algorithms used, i.e. SAW, MEW and M2EW, it is concluded that M2EW algorithm has better performance compare to SAW and MEW algorithms regarding RSD value. While in the same time M2EW has a lower delay average. The modification performed on MEW algorithm using Euclidean distance calculation has proven to increase the RSD value compare to MEW by $0.2 \%$ with the same delay value. Also, M2EW algorithm is proven to have performed better than SAW and MEW algorithms in case of background traffic class. For future work, we would like to implement the M2EW algorithm in another traffic class such as in streaming or conversation or interactive class.

\section{Acknowledgment}

Authors would like to thankfully acknowledge the resource support by School of Computing, Telkom University and Hardware and Embedded System Laboratory for this research work. 


\section{References}

[1] A. Shankar and J. Natarajan, "Base Station Positioning in Wireless Sensor Network to aid Cluster Head Selection Process", International Journal of Intelligent Engineering and Systems, Vol. 10, No. 2, pp. 173-182, 2017.

[2] T. Latha and L. Shakkera, "Towards Maximum Resource Utilization and Optimal Task Execution for Gaming IoT Workflow in Mobile Cloud", International Journal of Intelligent Engineering and Systems, Vol. 10, No. 1, pp. 134-143, 2017.

[3] E. Stevens-Navarro and V. W. Wong, "Comparison between vertical handoff decision algorithms for heterogeneous wireless networks", In: Proc. of the 2006 IEEE 63rd Vehicular Technology Conference, Vol. 2, pp. 947-951, 2006.

[4] M. Ravichandra, H. K. Gowda, and C. U. Kumar, "A survey on handovers literature for next generation wireless networks", International Journal of Advanced Research in Computer and Communication Engineering, Vol. 2, No. 12, pp.4671-4677, 2013.

[5] X. Yan, Y. A. Şekercioğlu, and S. Narayanan, "A survey of vertical handover decision algorithms in Fourth Generation heterogeneous wireless networks", Computer Networks, Vol. 54, No. 11, pp. 1848-1863, 2010.

[6] M. Drissi and M. Oumsis, "Performance evaluation of multi-criteria vertical handover for heterogeneous wireless networks", In: Proc. of the Intelligent Systems and Computer Vision, pp. $1-5,2015$.

[7] N. Gundluru and P. R. Ch, "Soft-Computing Based Trust Management Framework for Group Key Management in MANETs", International Journal of Intelligent Engineering and Systems, Vol. 10, No. 3, pp. 327-336, 2017.

[8] K. Savitha and C. Chandrasekar, "Vertical Handover decision schemes using SAW and WPM for Network selection in Heterogeneous Wireless Networks", ArXiv Prepr. ArXiv11094490, 2011.

[9] Z. A. Polgár, A. C. Hosu, Z. I. Kiss, and M. Varga, "Vertical Handover Decision Algorithm for Heterogeneous Cellular-WLAN Networks," MACRo 2015, Vol. 1, No. 1, pp. 1-12, 2015.

[10] S. Kunarak, "Vertical Handover Decision Based on RBF Approach for Ubiquitous Wireless Networks," In: Proc. of the 2016 International Conference on Platform Technology and Service, pp. 1-4, 2016.
[11] H. Breu, J. Gil, D. Kirkpatrick, and M. Werman, "Linear time Euclidean distance transform algorithms", IEEE Transactions Pattern Analysis and Machine Intelligence, Vol. 17, No. 5, pp. 529-533, 1995.

[12] Y. Gao, M. G. Ierapetritou, and F. J. Muzzio, "Determination of the confidence interval of the relative standard deviation using convolution", Journal of Pharmaceutical Innovation, Vol. 8, No. 2, pp. 72-82, 2013. 